\title{
Assimetrias regionais. Que diferenças nos estilos de vida e na satisfação com a vida dos adolescentes? Um estudo realizado em alunos do $3^{\circ}$ ciclo do Ensino Básico em Portugal
}

\author{
Regional asymmetries. What are the differences in lifestyles \\ and life satisfaction among adolescents? A study of students \\ of the $3^{\text {rd }}$ cycle of Basic Education in Portugal
}

Tiago Paupério Ferreira Vieira ${ }^{1}$

Nuno José Corte-Real Correia Alves ${ }^{2}$

Cláudia Salomé Lima Dias ${ }^{2}$

António Manuel Leal Ferreira Mendonça da Fonseca ${ }^{2}$

${ }^{1}$ Laboratório de Psicologia do Desporto, Faculdade de Desporto, Universidade do Porto. R. Dr. Plácido Costa 91. 4200-450 Porto Portugal.

tiago.pauperio@gmail.com ${ }^{2}$ Gabinete de Psicologia do Desporto, Faculdade de Desporto, Universidade do Porto.

\begin{abstract}
Based on the importance of behavior and life satisfaction for the health of young people and the considerable regional differences that exist in mainland Portugal, an investigation was conducted. It sought to verify differences in levels of the practice of sport, tobacco and alcohol consumption and life satisfaction among students of the 3rd cycle of basic education living in different inland/coastal and rural/urban areas. The sample consisted of 5624 adolescents, aged between 12 and 17 years. The instrument used for data collection was the "Inventory of Health-Related Behavior of Adolescents" and a translated version of "The Satisfaction With Life Scale". The results revealed that there are higher levels of smoking among young people living in urban and inland areas, higher levels of alcohol consumption among young people living in rural and inland areas and higher levels of life satisfaction among adolescents living in urban and coastal areas. Levels of sport were identical in all geographical areas. It was also found that there were significant differences in all variables of the analysis.
\end{abstract}

Key words Adolescents, Practice of sport, Smoking, Alcohol consumption, Satisfaction with life
Resumo Partindo da importância dos comportamentos e da satisfação com a vida para a saúde dos jovens e das consideráveis assimetrias regionais existentes no território de Portugal Continental, tínhamos como objetivo para esta investigação: verificar a existência de diferenças nos níveis de prática desportiva, de consumo de tabaco e de álcool, e de satisfação com a vida nos alunos do $3^{\circ}$ Ciclo do Ensino Básico, residentes em diferentes áreas, nomeadamente, interior/litoral e rural/ urbano. A amostra foi constituída por 5624 adolescentes, com idades entre 12 e 17 anos. O instrumento utilizado para a coleta dos dados foi o "Inventário de Comportamentos Relacionados com a Saúde dos Adolescentes" e uma versão traduzida e adaptada da "Satisfaction With Life Scale". Concluimos existirem níveis superiores de consumo de tabaco nos jovens residentes nas áreas interiores e urbanas, niveis superiores de consumo de álcool nos jovens residentes em áreas interiores e rurais, e niveis superiores de satisfação com a vida nos jovens residentes em áreas litorais e urbanas. Os niveis de prática desportiva foram idênticos em todos os locais de residência. Constatamos, também, a existência de diferenças significativas em todas as variáveis em análise.

Palavras-chave Adolescentes, Prática desportiva, Consumo de tabaco, Consumo de álcool, Satisfação com a vida 


\section{Introdução}

\section{Saúde e adolescência}

A saúde, tantas vezes ignorada, é um conceito essencial para as nossas vidas, enquanto maior "recurso" do ser humano. Entendendo saúde como "capacidade de criar e lutar por um projeto de vida pessoal e original em direção ao bem -estar"1,2, sabemos hoje que são diversas as suas determinantes e condicionantes que vão desde os sistemas de cuidados de saúde até o envolvimento físico, social e econômico, passando pela qualidade de vida, bem-estar e os estilos de vida ${ }^{3}$.

No entanto, a maioria das ameaças à saúde resultam de fatores comportamentais donde se destacam, de acordo com a Organização Mundial de Saúde, a inatividade física, os abusos de substâncias e os distúrbios alimentares ${ }^{4}$. Estes comportamentos de risco para a saúde, para além da influência direta no fenômeno saúde-doença, no curto prazo, têm, também, influência sobre os comportamentos futuros, pois vários estudos demonstram que o comportamento anterior constitui o principal preditor do comportamento futuro ${ }^{5}$.

Neste contexto, a adolescência, enquanto etapa do desenvolvimento humano, que pressupõe a passagem de uma situação de dependência infantil para a inserção social e a formação de um sistema de valores que definem a idade adulta ${ }^{6}$, caracterizada por rápidas alterações físicas, psicológicas, sócio-culturais e cognitivas, num trajeto que conduz à identidade e autonomia ${ }^{7}$, assume-se como fase fundamental na promoção da saúde, enquanto processo de habilitar as pessoas para melhorar e aumentar o controle sobre a sua saúde ${ }^{8}$ e na aquisição de comportamentos protetores da saúde.

\section{Comportamentos de risco e protetores da saúde dos adolescentes}

A atividade física, outrora ocupando parte substancial das rotinas diárias dos jovens, vem diminuindo substancialmente ao longo dos tempos, resultando em consequências graves em termos de saúde, pois esta progressiva inatividade tem sido, e é cada vez mais, associada a um vasto conjunto de patologias, nomeadamente às doenças cardiovasculares, diabetes, cancro e seus fatores de risco, como pressão arterial elevada, aumento do açúcar no sangue e sobrepeso, sendo, atualmente, apontada como o quarto principal fator de risco para a mortalidade global $-6 \%$ das mortes no mundo9 .
Apesar de todos os benefícios de ordem física e psicológica, descritos na literatura e associados a uma prática regular de atividade física, como o crescimento saudável, em termos físicos, nomeadamente na criação e manutenção de ossos, músculos e articulações saudáveis, controle de peso, redução da gordura e prevenção da hipertensão $\operatorname{arterial}^{10,11}$, e em termos psíquicos, aumentando o desempenho escolar ${ }^{12}$, por exemplo, enriquecendo o repertório psicomotor e ajudando a prevenir e controlar comportamentos de risco, como o tabaco, a violência, o álcool, a dependência a outras substâncias e a adesão a dietas pouco saudáveis ${ }^{10,13}$, os resultados de várias investigações apontam para uma reduzida prática desportiva dos jovens, de uma forma geral, e nos jovens do sexo feminino em particular, e para um decréscimo da mesma com a idade ${ }^{4,14-17}$.

Relativamente ao consumo de substâncias, o consumo de tabaco é a principal causa de doença e morte evitável, sendo responsável por cerca de $14 \%$ do total de mortes verificadas anualmente nos países desenvolvidos ${ }^{18}$. Nos jovens, o tabagismo ou o hábito de consumir tabaco é particularmente relevante pois, na maioria das vezes, a iniciação ocorre na puberdade e a dependência instala-se durante a adolescência ${ }^{19}$. Apesar do tabaco e fumar tabaco ter uma conotação negativa entre os jovens, incluindo os que fumam, principalmente nos jovens do sexo feminino ${ }^{19}$, das campanhas de prevenção do consumo de tabaco e da produção de leis que procuram reduzir o consumo e proteger os consumidores passivos, várias investigações apontam para significativas taxas de consumo idênticas entre sexos, e um aumento expressivo com a idade $e^{4,14,16,19,20}$.

Por outro lado, o consumo de álcool, com raízes nas civilizações antigas, constitui-se, na atualidade, como um dos mais sérios problemas sociais em escala mundial. Para além de ser, nos países desenvolvidos, uma das dez maiores causas de doença e ferimentos, responsável por cerca de 9\% de todos os problemas de saúde e por 3\% das mortes mundiais $-\approx 1,8$ milhões ${ }^{21}-$, estima-se que os custos do consumo, para a saúde, se situem entre os $2 \%$ e os $5 \%$ do Produto Interno Bruto dos países, tornando-se assim a redução do seu consumo uma das mais importantes ações de Saúde Pública, no sentido da promoção da saúde e recuperação econômica ${ }^{22}$.

No entanto, apesar de todos os malefícios e consequências do seu consumo, o álcool é uma substância legalizada, que se pode produzir e consumir livremente, sendo visto como um tipo especial de bem de consumo e não como uma 
substância que pode causar dependência ${ }^{22}$. Esta liberalização tem nos adolescentes especial relevância, pois o consumo de álcool surge muitas vezes como uma espécie de ritual de transição quase obrigatório, e o primeiro contacto destes com o álcool dá-se, na maioria das vezes, no seio da própria família e em casa ${ }^{23}$. Assim, o consumo de álcool entre a população adolescente é significativo, apontando as diversas investigações para consumos superiores no sexo masculino e nos jovens mais velhos ${ }^{4,14,18,24}$.

Por outro lado, as rápidas alterações, a multiplicidade de contextos sociais e interpessoais em que os adolescentes se movem e as variações na existência e no ritmo das relações influenciam, de igual forma, aspetos psicológicos no desenvolvimento dos jovens como a satisfação com a vida.

\section{Satisfação com a vida}

A satisfação com a vida, componente cognitiva de um constructo mais abrangente, o bem-estar subjetivo $^{25}$, pode ser entendida como o julgamento que os indivíduos fazem em relação à qualidade da sua própria vida com base nos seus próprios critérios ${ }^{26} \mathrm{e}$, atualmente, é vista como um conceito chave na investigação da "psicologia positiva", havendo razões para crer que se torna numa variável psicológica antes da idade adulta, mais particularmente no decurso da adolescência ${ }^{27}$.

Vários estudos associam elevados níveis de satisfação com a vida, nos adolescentes, com menor adoção de comportamentos de risco para a saúde, com melhor rendimento acadêmico, melhor relacionamento intra e interpessoal, menores índices de ansiedade e depressão e índices superiores de esperança e autocontrole ${ }^{28}$, pelo que esta pode tornar-se uma variável "chave" na identificação de diferentes estilos de vida e na promoção de estilos de vida saudáveis entre os jovens.

Por outro lado, tal como já referido, o envolvimento físico, social e econômico são, também, importantes e significativas determinantes da saúde ${ }^{3}$.

Neste contexto o local de residência poderá ter um papel significativo na saúde e na promoção desta nos jovens, bem como pode influenciar os seus estilos de vida e satisfação com a vida.

\section{Os locais de residência}

Portugal é um país dual e assimétrico. A complexa organização territorial, que remonta à década de 60 do século passado e ao processo de modernização que emergiu com alguma rele- vância nesses anos, resultou num país tantas vez apelidado de "país a duas velocidades", devido às enormes diferenças sociais, econômicas e territoriais entre as áreas que o constituem ${ }^{29}$.

A oposição entre grupos sociais, setores econômicos e espaços modernos e tradicionais introduziu no país vários contrastes, entre os quais o contraste entre o interior e o litoral e entre o rural e o urbano. O Portugal urbanizado, industrializado, infraestruturado e demograficamente dinâmico (característico das áreas litorais e urbanas) destaca-se, de forma inequívoca, do Portugal agrícola, subdesenvolvido, física e simbolicamente remoto e demograficamente repulsivo (característico das áreas interiores e rurais) ${ }^{30}$.

Em Portugal continental 50\% da população reside em zonas urbanas, $24 \%$ em zonas mediamente urbanas e $26 \%$ em zonas rurais ${ }^{30}$.

Neste contexto, atendendo às fortes diferenças territoriais existentes e conscientes que estas poderão, eventualmente, desempenhar um papel relevante nos níveis de prática desportiva, de consumo de tabaco e álcool e na satisfação com a vida dos adolescentes, tínhamos como objetivos para este estudo: verificar a existência de diferenças nos níveis de prática desportiva, de consumo de tabaco e de álcool e de satisfação com a vida nos alunos do $3^{\circ}$ Ciclo do Ensino Básico, residentes em diferentes áreas, nomeadamente, interior/ litoral e rural/urbano.

\section{Metodologia}

\section{Amostra}

A amostra deste estudo foi constituída por 5624 adolescentes $(53 \%$ q e $47 \%$ ○) ), com idades compreendidas entre os 12 e os 17 anos (média $=14,33 ; \sigma=1,359$ ), frequentadores do $3^{\circ}$ Ciclo do Ensino Básico em escolas de Portugal Continental.

Dividimos os alunos em grupos, de acordo com a sua área de residência, sob dois pontos de vista tradicionais de divisão imaginária do território português, a saber: litoral/interior e urbano/rural.

Relativamente à divisão litoral/interior, constituímos dois grupos: litoral $(55,2 \%)$ e interior $(44,8)$. Para a realização desta divisão e na ausência de uma delimitação institucional, recorremos ao padrão "clássico" referido à acessibilidade em relação à costa, preconizado por Estela Alegria ${ }^{31}$, classificando como áreas litorâneas as unidades que confinavam com o mar e aquelas cujo conce- 
lho "principal" (o de maior população em 1991) distavam menos de 60 quilômetros de Lisboa ou do Porto, e como do "Interior", as restantes.

No que diz respeito à divisão Urbano/Rural, constituímos dois grupos: Área Predominantemente Urbana - APU (75\%) - doravante designada por Área Urbana e Área Predominantemente Rural - APR (25\%) - doravante designada por Área Rural.

Optamos por excluir as Áreas Medianamente Urbanas (AMU) desta análise, por considerarmos que estas, por englobarem características comuns às Áreas Predominantemente Rurais (APR) e Áreas Predominantemente Urbanas (APU), poderiam enviesar os resultados obtidos.

Para a realização desta divisão, utilizamos as definições estabelecidas pelo Instituto Nacional de Estatística ${ }^{32,33}$, as quais têm por base o peso da população residente na população total das pequenas povoações e o peso da área na área total das pequenas povoações.

\section{Procedimentos}

O instrumento utilizado para a coleta dos dados foi o "inventário de comportamentos relacionados com a saúde dos adolescentes", desenvolvido por Corte-Real et al. ${ }^{34} \mathrm{e}$ a "Escala de Satisfação com a Vida", versão traduzida e adaptada da "Satisfaction With Life Scale" (SWLS) de Diener et al..$^{35}$.

Os dados foram recolhidos entre 2002 e 2008 nas escolas de Portugal Continental em que a investigação foi aprovada pelo Conselho Pedagógico e pelo Conselho Executivo (35 escolas), na presença de um investigador do Laboratório de Psicologia do Desporto da Faculdade de Desporto da Universidade do Porto, que acompanhou e monitorizou a aplicação do instrumento de coleta de dados.

O estudo foi aprovado, no início, pelo Conselho Científico da Faculdade de Desporto da Universidade do Porto, entidade responsável por assegurar o cumprimento da Declaração de Helsínque em estudos humanos e do Ministério da Educação Português.

$\mathrm{Na}$ análise dos dados, utilizamos estatística descritiva com a apresentação das frequências e percentagens para as variáveis nominais, médias e desvio padrão para as variáveis contínuas. Recorremos, ainda, ao teste Qui-Quadrado (estudo da distribuição em variáveis nominais) com análise de residuais ajustados (para localização de valores significativos). O nível de significância considerado foi de 0,05 , sendo apresentado em negrito, nos quadros, os valores com residuais ajustados iguais ou superiores a 1,9, em cada célula.

\section{Variáveis}

Para além das variáveis, já referenciadas, sexo, escalão etário e local de residência, utilizamos ainda as seguintes variáveis: prática desportiva (PD), consumo de tabaco (CT), consumo de álcool (CA) e a satisfação com a vida (SV).

Para cada uma das variáveis a constituição dos grupos foi realizada de acordo com o quadro seguinte (Quadro 1).

\section{Resultados}

\section{Análise da amostra global}

Iniciando a apresentação dos resultados por uma análise à amostra global, independente dos meios de residência, verificamos que apenas $38 \%$ dos jovens praticavam esportes com regularidade (mais de três vezes por semana), 36\% tinham uma prática reduzida (até 3 vezes por semana) e que $26 \%$ nunca praticavam ou praticavam menos de uma vez por semana.

Relativamente ao consumo de tabaco, registramos que 7\% dos jovens da nossa amostra consumiam regularmente tabaco (diariamente), $8 \%$ consumiam de forma reduzida ou esporádica (até várias vezes por semana) e $85 \%$ não consumiam.

No que diz respeito ao consumo de álcool, verificamos que $6 \%$ dos jovens consumia regularmente álcool, $25 \%$ consumia de forma reduzida ou esporádica (até uma vez por semana) e 69\% não consumia.

Por fim e relativamente à satisfação com a vida, verificamos que $54 \%$ dos jovens da amostra consideravam-se muito satisfeitos com a sua vida, 36\% moderadamente satisfeitos e $10 \%$ pouco satisfeitos.

\section{Análise dos resultados em função do local de residência interior/litoral}

Verificámos, na amostra global, (Tabela 1) existirem diferenças estatisticamente significativas em todas as variáveis analisadas, exceto na prática desportiva. Assim, os jovens residentes no interior registravam consumos de tabaco e de álcool superiores aos dos jovens residentes em litoral, e níveis inferiores de satisfação com a vida. 


\begin{tabular}{|c|c|c|c|}
\hline Variável & Grupo & Critérios de inclusão & Observações \\
\hline $\mathrm{PD}$ & $\begin{array}{l}\text { Inexistente ou esporádica } \\
\text { Reduzida } \\
\text { Regular }\end{array}$ & $\begin{array}{l}\text { Não pratica/ pratica até uma vez por } \\
\text { semana. } \\
\text { Pratica até três vezes por semana. } \\
\text { Pratica mais de três vezes por } \\
\text { semana. }\end{array}$ & $\begin{array}{l}\text { Analisámos a frequência de } \\
\text { prática igual ou superior a } 1 \text { hora } \\
\text { por sessão. Considerámos apenas } \\
\text { a PD, quer de âmbito recreativo } \\
\text { quer de âmbito competitivo, } \\
\text { realizada fora da escola. }\end{array}$ \\
\hline CT & $\begin{array}{l}\text { Regular } \\
\text { Reduzido } \\
\text { Inexistente }\end{array}$ & $\begin{array}{l}\text { Consome diariamente } \\
\text { Consome esporadicamente (até } \\
\text { várias vezes por semana) } \\
\text { Não consome }\end{array}$ & $\begin{array}{l}\text { Analisamos a frequência de } \\
\text { consumo. }\end{array}$ \\
\hline CA & $\begin{array}{l}\text { Regular } \\
\text { Reduzido } \\
\text { Inexistente }\end{array}$ & $\begin{array}{l}\text { Consome várias vezes por semana } \\
\text { Consome até uma vez por semana } \\
\text { Não consome/quase nunca consome }\end{array}$ & $\begin{array}{l}\text { Analisamos a frequência de } \\
\text { consumo. }\end{array}$ \\
\hline SV & $\begin{array}{l}\text { Reduzida } \\
\text { Moderada } \\
\text { Elevada }\end{array}$ & $\begin{array}{l}(\bar{X} \text { entre } 1 \text { e } 2,4) \\
(\bar{X} \text { entre } 2,5 \text { e } 3,4) \\
(\bar{X} \text { entre } 3,5 \text { e } 5)\end{array}$ & $\begin{array}{l}\text { Determinámos o valor médio } \\
\text { tendo em conta a resposta a } \\
\text { cinco questões com valores entre } \\
1 \text { (discordo totalmente) e } 5 \\
\text { (concordo totalmente) }\end{array}$ \\
\hline
\end{tabular}

Tabela 1. Análise da prática desportiva, consumo de tabaco, consumo de álcool e satisfação com a vida em função do meio (litoral e interior) na amostra total e em cada sexo.

\begin{tabular}{|c|c|c|c|c|c|c|c|}
\hline & & \multicolumn{2}{|c|}{$\begin{array}{c}\text { Amostra Global } \\
(5624)\end{array}$} & \multicolumn{2}{|c|}{$\begin{array}{l}\text { Sexo Feminino }+ \\
\quad(n=2964)\end{array}$} & \multicolumn{2}{|c|}{$\begin{array}{l}\text { Sexo Masculino } \bar{\lambda} \\
\quad(n=2660)\end{array}$} \\
\hline & & $\begin{array}{c}\text { Litoral } \\
(\mathbf{n}=3105)\end{array}$ & $\begin{array}{l}\text { Interior } \\
(n=2519)\end{array}$ & $\begin{array}{c}\text { Litoral } \\
(\mathrm{n}=1683)\end{array}$ & $\begin{array}{c}\text { Interior } \\
(n=1281)\end{array}$ & $\begin{array}{c}\text { Litoral } \\
(\mathrm{n}=1422)\end{array}$ & $\begin{array}{c}\text { Interior } \\
(n=1238)\end{array}$ \\
\hline \multicolumn{8}{|l|}{ PD } \\
\hline Inexistente ou esporádica & & 27 & 26 & 34 & 32 & 18 & 20 \\
\hline Reduzida & \multirow{3}{*}{$\%$} & 36 & 35 & 39 & 37 & 33 & 33 \\
\hline Regular & & 37 & 39 & 27 & 31 & 49 & 47 \\
\hline$($ g.l. $=2)$ & & $\chi^{2}=1,402$ & $\mathrm{p}=0,496$ & $\chi^{2}=3,410$ & $\mathrm{p}=0,182$ & $\chi^{2}=2,104$ & $\mathrm{p}=0,349$ \\
\hline \multicolumn{8}{|l|}{ CT } \\
\hline Regular & \multirow{4}{*}{$\%$} & 6 & 10 & 6 & 8 & 5 & 12 \\
\hline Reduzido & & 7 & 9 & 7 & 8 & 6 & 10 \\
\hline Inexistente & & 87 & 81 & 87 & 84 & 89 & 78 \\
\hline$($ g.l. $=2)$ & & $\chi^{2}=45,958$ & $\mathrm{p}<0,001$ & $\chi^{2}=4,196$ & $\mathrm{p}=0,123$ & $\chi^{2}=59,381$ & $\mathrm{p}<0,001$ \\
\hline \multicolumn{8}{|c|}{ 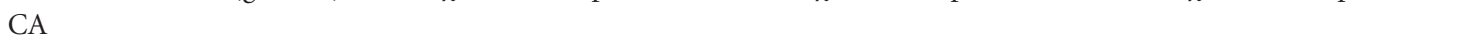 } \\
\hline Regular & \multirow{3}{*}{$\%$} & 5 & 9 & 3 & 4 & 7 & 14 \\
\hline Reduzido & & 23 & 26 & 23 & 26 & 23 & 26 \\
\hline Inexistente & & 72 & 65 & 74 & 70 & 70 & 60 \\
\hline$($ g.l. $=2)$ & & $\chi^{2}=47,186$ & $\mathrm{p}<0,001$ & $\chi^{2}=4,046$ & $\mathrm{p}=0,132$ & $\chi^{2}=25,824$ & $\mathrm{p}<0,001$ \\
\hline \multicolumn{8}{|l|}{ SV } \\
\hline Reduzida & \multirow{3}{*}{$\%$} & 10 & 11 & 11 & 13 & 9 & 9 \\
\hline Moderada & & 34 & 38 & 36 & 37 & 32 & 39 \\
\hline \multirow[t]{2}{*}{ Elevada } & & 56 & 51 & 53 & 50 & 59 & 52 \\
\hline & & $\chi^{2}=14,604$ & $\mathrm{p}=0,001$ & $\chi^{2}=2,782$ & $\mathrm{p}=0,249$ & $\chi^{2}=17,463$ & $\mathrm{p}<0,001$ \\
\hline
\end{tabular}


Por fim e relativamente à prática desportiva, ainda que não tenhamos encontrado diferenças estatisticamente significativas, registramos a tendência para os níveis de prática regulares serem superiores no interior.

Seguidamente, procedemos a uma análise por sexo em cada local de residência em estudo (Tabela 1) verificando que, de uma forma geral, parecem ser os rapazes os mais influenciados pelo local de residência interior/litoral, visto que em todas as variáveis em estudo, exceto na prática desportiva, as maiores diferenças entre locais, encontravam-se no sexo masculino existindo, inclusive, diferenças estatisticamente significativas.

Por outro lado, a análise por sexos permitiunos confirmar o que já havíamos descrito quando da análise da amostra global: os alunos residentes no interior apresentavam consumos superiores de tabaco e de álcool e índices mais reduzidos de satisfação com a vida, quando comparados com os alunos residentes no litoral, em ambos os sexos.

Exceção à regra, apenas a prática desportiva no sexo feminino os níveis inferiores de prática revelaram-se nos alunos residentes no litoral, enquanto que, no sexo masculino, estes, registaramse nos alunos residentes no interior.
Posto isto, e no intuito de perceber se existiriam escalões etários eventualmente mais influenciados pelos diferentes locais de residência, optamos por realizar uma análise por local em cada escalão etário (Tabela 2). Verificamos existirem diferenças estatisticamente significativas no consumo de tabaco em todos os escalões etários, no Consumo de Álcool no escalão etário de 1617 anos e na satisfação com a vida no escalão etário de 12-13 anos.

Assim, e no que diz respeito ao consumo de tabaco, constatamos, como já havíamos feito na análise da amostra global, existirem consumos superiores nos jovens residentes no interior $\mathrm{e}$ verificamos que tal fato era válido para todos os escalões etários.

Relativamente ao consumo de álcool, verificamos que era consideravelmente superior nos jovens residentes no interior em todos os escalões etários, principalmente no escalão etário de 16-17 anos, onde se registou uma diferença estatisticamente significativa.

No que concerne à satisfação com a vida constatamos existirem níveis mais elevados nos jovens residentes no litoral, em todos os escalões etários, registando-se, inclusive, diferença estatis-

Tabela 2. Análise da prática desportiva, consumo de tabaco, consumo de álcool e satisfação com a vida em função do meio (litoral e interior), em cada escalão etário.

\begin{tabular}{|c|c|c|c|c|c|c|c|}
\hline & & \multicolumn{2}{|c|}{$\begin{array}{l}12-13 \text { anos } \\
(\mathrm{n}=1694)\end{array}$} & \multicolumn{2}{|c|}{$\begin{array}{c}14-15 \text { anos } \\
(\mathrm{n}=2802)\end{array}$} & \multicolumn{2}{|c|}{$\begin{array}{l}16-17 \text { anos } \\
(n=1128)\end{array}$} \\
\hline & & $\begin{array}{c}\text { Litoral } \\
(\mathrm{n}=1102)\end{array}$ & $\begin{array}{l}\text { Interior } \\
(\mathrm{n}=592)\end{array}$ & $\begin{array}{c}\text { Litoral } \\
(\mathrm{n}=1491)\end{array}$ & $\begin{array}{c}\text { Interior } \\
(\mathrm{n}=1311)\end{array}$ & $\begin{array}{c}\text { Litoral } \\
(\mathrm{n}=512)\end{array}$ & $\begin{array}{c}\text { Interior } \\
(n=616)\end{array}$ \\
\hline \multicolumn{8}{|l|}{$\mathrm{PD}$} \\
\hline Inexistente o & esporádica & 26 & 24 & 25 & 27 & 32 & 27 \\
\hline Reduzida & $\%$ & 36 & 34 & 37 & 35 & 35 & 37 \\
\hline Regular & $\%$ & 38 & 42 & 38 & 38 & 33 & 36 \\
\hline & $(\mathrm{g} .1 .=2)$ & $\chi^{2}=2,129$ & $\mathrm{p}=0,345$ & $\chi^{2}=1,384$ & $\mathrm{p}=0,501$ & \multicolumn{2}{|c|}{$\chi^{2}=3,716 p=0,156$} \\
\hline \multicolumn{8}{|l|}{ CT } \\
\hline Regular & & 2 & 5 & 5 & 6 & 16 & 22 \\
\hline Reduzido & $\%$ & 4 & 5 & 8 & 10 & 9 & 11 \\
\hline Inexistente & $\%$ & 94 & 90 & 87 & 84 & 75 & 67 \\
\hline & $($ g.l. $=2)$ & $\chi^{2}=12,156$ & $\mathrm{p}=0,002$ & $\chi^{2}=7,112$ & $\mathrm{p}=0,029$ & $\chi^{2}=7,233$ & $\mathrm{p}=0,027$ \\
\hline \multicolumn{8}{|l|}{ CA } \\
\hline Regular & $\%$ & 3 & 5 & 4 & 5 & 8 & 19 \\
\hline Reduzido & & 20 & 20 & 22 & 24 & 34 & 36 \\
\hline Inexistente & & 77 & 75 & 74 & 71 & 58 & 45 \\
\hline & $($ g.l. $=2)$ & $\chi^{2}=2,082$ & $\mathrm{p}=0,353$ & $\chi^{2}=2,849$ & $\mathrm{p}=0,241$ & $\chi^{2}=37,748$ & $\mathrm{p}<0,001$ \\
\hline \multicolumn{8}{|l|}{ SV } \\
\hline Reduzida & $\%$ & 8 & 8 & 11 & 9 & 16 & 16 \\
\hline Moderada & $\%$ & 30 & 39 & 35 & 38 & 36 & 40 \\
\hline \multirow[t]{2}{*}{ Elevada } & & 62 & 53 & 54 & 53 & \multirow{2}{*}{\multicolumn{2}{|c|}{$\chi^{2}=2,355 p=0,308$}} \\
\hline & $($ g.l. $=2)$ & $\chi^{2}=12,361$ & $\mathrm{p}=0,002$ & $\chi^{2}=1,758$ & $\mathrm{p}=0,415$ & & \\
\hline
\end{tabular}


ticamente significativa no escalão etário de 12-13 anos.

Por fim e relativamente à prática desportiva, tal como já referimos, não encontramos qualquer diferença estatisticamente significativa em qualquer escalão etário analisado. Não obstante, a análise sugere a existência de níveis mais frequentes de prática nos jovens residentes no interior nos escalões etários de 12-13 anos e 16-17 anos, e níveis iguais no escalão etário de 14-15 anos.

\section{Análise dos resultados em função do meio de residência rural/urbano}

Após a análise por local de residência interior/litoral, procedemos a análise das diferenças entre jovens residentes em Áreas Rurais (AR) e Áreas Urbanas (AU).

Assim, e relativamente à amostra global, verificamos existirem diferenças, estatisticamente significativas, em todas as variáveis, exceto na variável prática desportiva (Tabela 3 ). Constatamos a existência de níveis superiores de consumo de tabaco e de satisfação com a vida do meio urbano e níveis superiores de consumo de álcool no meio rural. Relativamente à prática desportiva verificamos que os valores de prática eram muito idênticos em ambos os meios em análise.

Seguidamente e tal como já havíamos feito, quando da análise por meio interior/litoral, realizamos uma análise por local em cada um dos sexos e verificamos existirem diferenças estatisticamente significativas na satisfação com a vida no sexo feminino e no consumo de tabaco e consumo de álcool no sexo masculino (Tabela 3).

Assim e no que diz respeito à satisfação com a vida no sexo feminino, verificámos existirem níveis mais elevados nas jovens residentes em meio urbano, quando comparadas com as residentes em meio rural. Nas restantes variáveis, no sexo feminino, e não obstante a inexistência de diferenças estatisticamente significativas, registramos que a prática desportiva era ligeiramente superior no meio rural e que os consumos de tabaco e de álcool tendiam a ser superiores no meio urbano, em especial o consumo de tabaco.

Já relativamente ao sexo masculino e ao consumo de tabaco, verificamos que, embora as percentagens de consumo regular fossem iguais entre os meios de residência em análise, as percentagens de consumo inexistentes eram subs-

Tabela 3. Análise da prática desportiva, consumo de tabaco, consumo de álcool e satisfação com a vida em função do meio (urbano e rural) na amostra total e em cada sexo.

\begin{tabular}{|c|c|c|c|c|c|c|c|}
\hline & & \multicolumn{2}{|c|}{$\begin{array}{c}\text { Amostra Global } \\
(4592)\end{array}$} & \multicolumn{2}{|c|}{$\begin{array}{l}\text { Sexo Feminino } q \\
\quad(n=2430)\end{array}$} & \multicolumn{2}{|c|}{$\begin{array}{l}\text { Sexo Masculino } \\
\quad(n=2660)\end{array}$} \\
\hline & & $\begin{array}{c}\text { Urbano } \\
(\mathrm{n}=3442)\end{array}$ & $\begin{array}{c}\text { Rural } \\
(n=1150)\end{array}$ & $\begin{array}{c}\text { Urbano } \\
(\mathrm{n}=1862)\end{array}$ & $\begin{array}{c}\text { Rural } \\
(n=568)\end{array}$ & $\begin{array}{c}\text { Urbano } \\
(n=1580)\end{array}$ & $\begin{array}{c}\text { Rural } \\
(n=582)\end{array}$ \\
\hline \multicolumn{8}{|l|}{$\mathrm{PD}$} \\
\hline Inexistente ou esporádica & \multirow{4}{*}{$\%$} & 27 & 27 & 33 & 32 & 18 & 22 \\
\hline Reduzida & & 36 & 34 & 38 & 37 & 34 & 31 \\
\hline Regular & & 37 & 39 & 29 & 31 & 48 & 47 \\
\hline$($ g.l. $=2)$ & & \multicolumn{2}{|c|}{$\chi^{2}=1,074 p=0,584$} & \multicolumn{2}{|c|}{$\chi^{2}=0,928 \mathrm{p}=0,629$} & \multicolumn{2}{|c|}{$\chi^{2}=3,269 \mathrm{p}=0,195$} \\
\hline \multicolumn{8}{|l|}{ CT } \\
\hline Regular & \multirow{4}{*}{$\%$} & 8 & 7 & 8 & 5 & 9 & 9 \\
\hline Reduzido & & 7 & 9 & 7 & 7 & 6 & 11 \\
\hline Inexistente & & 85 & 84 & 85 & 88 & - & 80 \\
\hline$($ g.l. $=2)$ & & $\chi^{2}=9,822$ & $\mathrm{p}=0,007$ & $\chi^{2}=5,723$ & $\mathrm{p}=0,057$ & $\chi^{2}=14,65$ & $\mathrm{p}=0,001$ \\
\hline \multicolumn{8}{|c|}{$\pi$} \\
\hline Regular & \multirow{3}{*}{$\%$} & 6 & 8 & 3 & 2 & 9 & 13 \\
\hline Reduzido & & 23 & 27 & 23 & 26 & 23 & 28 \\
\hline Inexistente & & 71 & 65 & 74 & 72 & 68 & 59 \\
\hline$($ g.l. $=2)$ & & $\chi^{2}=10,139$ & $\mathrm{p}<0,001$ & $\chi^{2}=3,296$ & $\mathrm{p}=0,192$ & $\chi^{2}=19,57$ & $\mathrm{p}<0,001$ \\
\hline \multicolumn{8}{|l|}{ SV } \\
\hline Reduzida & \multirow{3}{*}{$\%$} & 11 & 10 & 12 & 11 & 9 & 8 \\
\hline Moderada & & 34 & 41 & 35 & 43 & 34 & 39 \\
\hline \multirow[t]{2}{*}{ Elevada } & & 55 & 49 & 53 & 46 & 57 & 53 \\
\hline & & $\chi^{2}=16,479$ & $\mathrm{p}<0,001$ & $\chi^{2}=12,20$ & $\mathrm{p}=0,002$ & $\chi^{2}=5,753$ & $\mathrm{p}=0,056$ \\
\hline
\end{tabular}


tancialmente maiores nos residentes no meio urbano. No que diz respeito ao consumo de álcool, constatamos a existência de consumos superiores nos rapazes residentes em meio rural.

Por fim e relativamente à prática desportiva e à satisfação com vida, embora não tivéssemos encontrado diferenças estatisticamente significativas, verificamos existirem níveis ligeiramente superiores de prática desportiva e de satisfação com a vida nos rapazes residentes em meio urbano.

Seguidamente, optámos, tal como também havíamos feito na análise por meio interior e litoral, por realizar uma análise por meio em cada escalão etário (Tabela 4).

Verificámos existirem diferenças estatisticamente significativas na satisfação com a vida no escalão etário de 12-13 anos e no consumo de tabaco e álcool no escalão etário de 16-17 anos. Para além disso, encontramos, também, algumas tendências dignas de registro.

Assim, verificamos a existência de valores ligeiramente superiores de prática desportiva no meio rural nos escalões etários de 12-13 anos e 14-15 anos e, no meio urbano, no escalão etário de 16-17 anos.
Constatamos, ainda, a existência de consumos de tabaco idênticos no escalão etário de 12-13 anos e valores superiores no meio urbano nos escalóes etários de 14-15 anos e 16-17 anos, verificando-se, neste último, diferenças estatisticamente significativas.

Relativamente ao consumo de álcool, verificamos existirem valores de consumo idênticos entre os meios de residência nos escalões etários de 12-13 anos e de 14-15 anos, e valores superiores no meio urbano no escalão etário de 16-17 anos (diferença estatisticamente significativa).

Por fim e relativamente à satisfação com a vida encontramos valores superiores no meio urbano em todos os escalões etário e, em particular, no escalão etário de 12-13 anos, onde a diferença era estatisticamente significativa.

\section{Discussão}

No decorrer desta investigação foram poucos os estudos encontrados com referência a diferenças nos comportamentos relacionados com a saúde ou na satisfação com a vida dos adolescentes, em diferentes meios de residência.

Tabela 4. Análise da prática desportiva, consumo de tabaco, consumo de álcool e satisfação com a vida em função do meio (urbano e rural) em cada escalão etário.

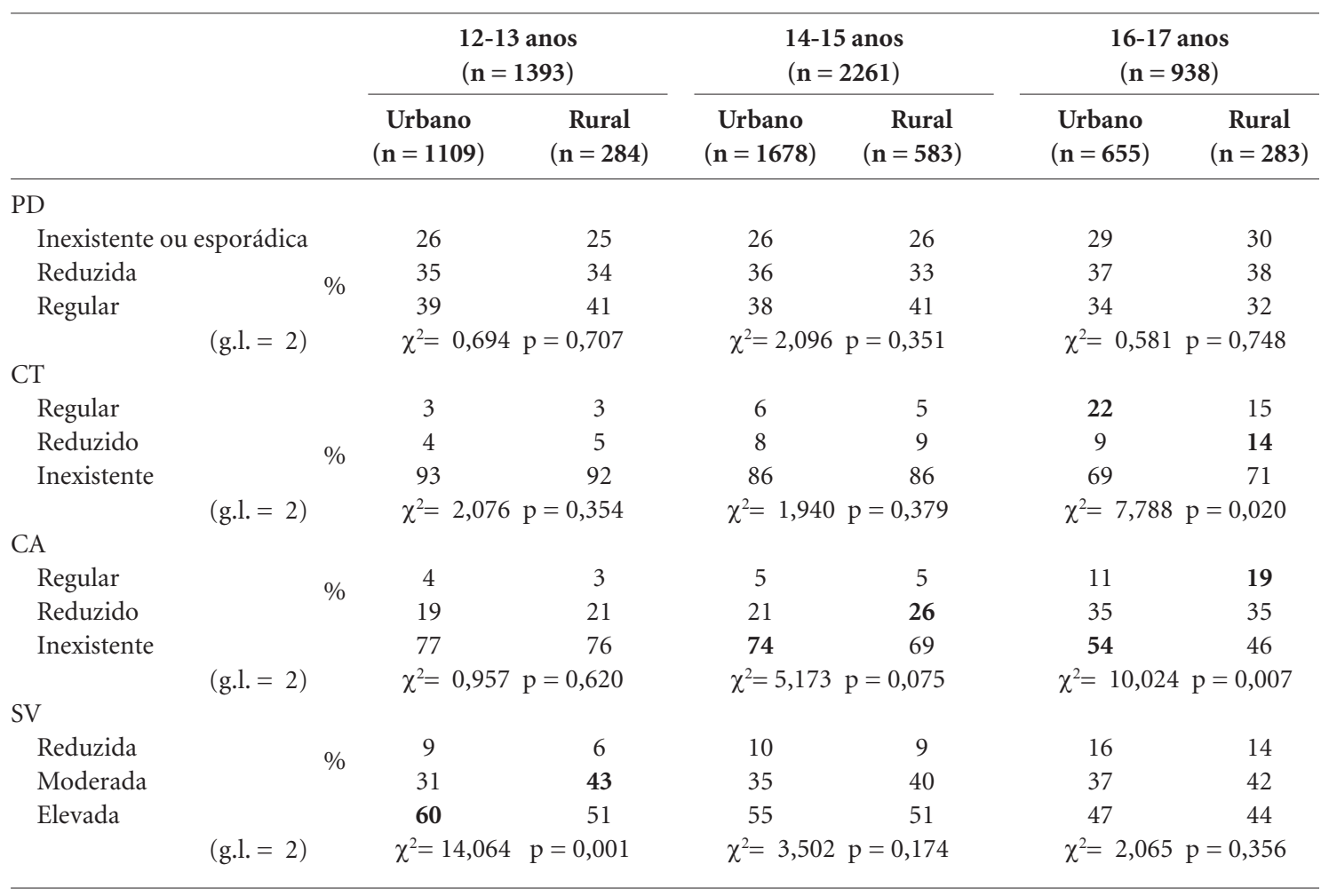


Não obstante, cremos que a análise de investigações, na mesma área de intervenção e em áreas adjacentes, possibilita discutir os resultados aqui encontrados.

Assim, e pela análise do estudo de Santana et $a .^{36}$, sobre a influência das regiões no estado de saúde da população portuguesa, verificamos existir uma variação do estado de saúde, na razão direta da urbanização/litoralização dos municípios, em dois períodos de análise distintos, resultado de um território marcado por fortes contrastes sociais, econômicos e demográficos devido à atração continuada da população ao litoral e à repulsa pelo interior que atrai ainda mais desequilíbrios.

Por outro lado, o mesmo estudo concluiu que as causas das desigualdades em saúde estão em estreita dependência de fatores econômicos e do meio físico e social que fazem parte do ambiente diário das pessoas (rendimento, habitação, desemprego, etc.).

Por outro lado e analisando o estudo de Youngblade e Curry ${ }^{37}$, constatamos que os comportamentos relacionados com a saúde sofrem influência direta dos contextos interpessoais em que os jovens e adolescentes vivem. Os resultados deste estudo sugeriram que jovens envolvidos em contextos que forneciam recursos positivos eram menos propensos à aquisição de comportamentos de risco e mais propensos ao desenvolvimento de comportamentos protetores da saúde.

Neste contexto, consideramos ser possível que os fortes contrastes sociais, econômicos e demográficos tenham influência significativa nos comportamentos relacionados com a saúde dos adolescentes, bem como na satisfação com a vida destes.

Posto isto e regressando à nossa investigação, mais concretamente à prática desportiva, verificamos existirem reduzidos níveis de prática desportiva regular, no geral, e nos jovens do sexo feminino e nos mais velhos, em particular.

Estes resultados convergem com os resultados registrados noutras investigações, nomeadamente nos estudos de Corte-Real et al. ${ }^{14}$, Currie et al. ${ }^{4}$, Matos et al. ${ }^{16}$ e Mota e Sallis ${ }^{17}$. Ainda relativamente à prática desportiva, constatamos não existirem diferenças nos níveis de prática desportiva nos jovens que residem nos meios em análise.

Assim e apesar de autores como Yang et al. (in Mota e Sallis ${ }^{17}$ ) referirem a possibilidade de populações mais urbanas terem mais escolhas e mais informações em relação ao exercício físico e maiores oportunidades de utilizar espaços e equipamentos desportivos e, por conseguin- te, apresentarem níveis superiores de prática, os nossos resultados sugerem que a prática desportiva dos jovens não é influenciada pela sua área de residência.

Cremos, pois, que a adesão a estilos de vida mais ativos não se prende tanto a fatores extrínsecos que tenham por base as questões de igualdades ou desigualdades oriundas dos diferentes meios de residência, como o estatuto socioeconómico ${ }^{38}$, mas sim a fatores de ordem intrínseca como o divertimento, o prazer e as competências tal como referido por Weinberg et al., Longhunst e Spink (in Mota e Sallis ${ }^{17}$ ) ou à influência dos pais ${ }^{38}$.

Relativamente ao consumo de tabaco, a literatura por nós consultada é unânime na referência a taxas não negligenciáveis de consumo entre os jovens, em particular nos mais velhos e idênticas entre os sexos ${ }^{4,14,16,19,20}$. Também no nosso estudo constatamos esses resultados. Para além disso, verificamos que os consumos eram significativamente superiores nos meios interior e urbano.

Resultados semelhantes foram encontrados por Matos et al. ${ }^{39}$ relativamente à maior prevalência de consumo de tabaco pelos jovens residentes no meio interior e por Fraga et al..$^{40}$, num estudo de revisão, relativamente à maior prevalência de consumo de tabaco pelos jovens residentes no meio urbano.

No entanto, poder-se-á equacionar a "força" da influência do meio de residência na adoção de hábitos de consumo desta substância por parte dos jovens, tendo em conta que vários estudos qualitativos sugerem que a iniciação tabágica ocorre no contexto do grupo de pares ${ }^{19}$ e que os principais fatores promotores do consumo são: algum dos progenitores fumar ou ter fumado, existir alguma pessoa em casa que fume, os irmãos mais velhos fumarem e os amigos fumarem ${ }^{41}$.

Os fatores apresentados e descritos nos estudos anteriores são transversais aos meios, em análise neste estudo, e não influenciados pela heterogeneidade cultural, econômica e social existente entre estes.

No que diz respeito ao consumo de álcool, o nosso estudo revelou significativas taxas de consumo, principalmente nos jovens mais velhos e nos jovens do sexo masculino. Resultados idênticos foram encontrados por Corte-Real et al. ${ }^{14}$, Currie et al. ${ }^{4}$, Granville-Garcia et al. ${ }^{24}$, Matos et al. ${ }^{16}$ e pelo Institute of Alcohol Studies ${ }^{21}$. Para além disso, verificamos que os consumos eram significativamente superiores nos meios interior e rural.

A este respeito encontramos apenas um estu$\mathrm{do}^{42}$ com referência a diferenças no consumo de 
álcool em jovens entre o meio interior (no caso, Beira Interior) e meio litoral (no caso, Grande Lisboa), onde se concluiu não existirem diferenças estatisticamente significativas entre os meios, ainda que os resultados sugerissem a existência de consumos ligeiramente superiores nos jovens residentes na área da grande Lisboa, o que diverge dos resultados por nós encontrados.

No entanto, cremos que os resultados por nós encontrados refletem questões culturais no sentido em que, tal como afirmamos no capítulo introdutório deste trabalho, "o consumo de álcool surge muitas vezes como uma espécie de ritual de transição quase obrigatório e o primeiro contacto dos jovens com o álcool dá-se na maioria das vezes no seio da própria família e em casa". Questões essas, possivelmente mais vincadas em sociedades/regiões agrícolas subdesenvolvidas, física e simbolicamente remotas, características das áreas interiores e rurais.

Por fim e relativamente à satisfação com a vida, os estudos por nós consultados apontavam para níveis de satisfação com a vida de uma forma geral elevados, em particular nos rapazes e nos mais jovens ${ }^{4,16,43}$. Os resultados por nós encontrados refletem exatamente a mesma realidade. Por outro lado, registramos, também, níveis superiores de satisfação com a vida nos meios litoral e urbano.

$\mathrm{Na}$ falta de estudos que nos permitam a comparação e discussão de resultados, cremos que as diferenças registradas no nosso estudo ficarão a dever às diferenças entre meios, no que aos processos de modernização diz respeito, ou seja, os níveis mais elevados de satisfação com a vida característica dos meios litorais e urbanos poderão estar relacionados com o fato destes meios serem mais urbanizados, industrializados, infraestruturados, demograficamente dinâmicos e socioeconomicamente mais desenvolvidos.

\section{Conclusões e sugestões}

Após a análise e discussão dos resultados, apesar da necessidade de mais estudos que confirmem ou não os resultados aqui encontrados, nomeadamente no que diz respeito à relação entre o local de residência, os comportamentos e a satisfação com a vida dos adolescentes portugueses, cremos que esta variável, o local de residência, não sendo com certeza única, nem porventura a mais importante, poderá estar intimamente relacionada com a adoção de comportamentos protetores ou de risco para a saúde e com a promoção de níveis mais elevados de satisfação com a vida, podendo-se assim falar da existência de locais mais ou menos protetores da saúde dos jovens e adolescentes.

Neste contexto, acreditamos que as enormes diferenças sociais, econômicas, culturais e demográficas, consequência de uma complexa organização territorial, existentes em diferentes locais de residência, promovem diferenças nos comportamentos e na satisfação dos adolescentes portugueses.

Assim, concluímos que, genericamente, as áreas rurais e interiores, pelas suas caraterísticas próprias, promovem uma maior adoção de comportamentos de risco, por parte dos adolescentes, mais significativa no sexo masculino e transversal a todos os escalões etários e níveis inferiores de satisfação com a vida, relativamente às áreas urbanas e litorais.

Perante isto, cientes dos custos financeiros da prevenção mas crentes de que esta é a melhor de todas as ferramentas de promoção da saúde, pensamos ser fundamental reforçar os programas de promoção da saúde existentes em Portugal, como o Plano Nacional de Saúde 2012-2016 ${ }^{44}$ ou o Programa Nacional de Saúde Escolar ${ }^{45}$, mais direcionado aos jovens e adolescentes, bem como à real adequação destes aos diferentes sexos, idades, particularidades e especificidades regionais.

Por fim, pensa mos, também, que o desenvolvimento e aplicação de programas de promoção da saúde, deverão estar baseados em resultados de investigações realizadas em cada realidade e não de dados médios nacionais, por forma a ajustarem-se às diferentes especificidades existentes no país, adequando, assim, a intervenção à realidade local. 


\section{Colaboradores}

T Paupério participou da conceção, pesquisa, metodologia e redação final do trabalho e NJ Corte-Real da conceção, metodologia e redação final do trabalho. CS Dias e AM Fonseca participaram da conceção e revisão final do trabalho.

\section{Referências}

1. Pestana C. Comportamentos de saúde em jovens em idade escolar - nota introdutória. In: Matos $\mathrm{M}$, equipa do Projecto Aventura Social \& saúde. A saúde dos adolescentes portugueses (Quatro anos depois). Lisboa: Edições FMH; 2003. p. 5.

2. Navarro F. Educar para a saúde ou para a Vida? Conceitos e fundamentos para novas práticas. In: Precioso J, Viseu F, Dourado L, Vilaça M, Henriques R, Lacerda T, organizadores. Educação para a saúde. Braga: Departamento de Metodologias da Educação da Universidade do Minho; 1999. p. 13-22.

3. Arah O, Westert G, Hurst J, Klazinga N. A conceptual framework for the OECD: Health Care Quality Indicators Project. Int J Qual Health Care 2006; 18:5-13.

4. Currie C, Gabhainn S, Godeau E, Roberts C, Smith R, Currie D, Picket W, Richter M, Morgan A, Barnekow V. Inequalities in young people's health - HBSC International Report from the 2005/2006 survey. Copenhagen: World Health Organization (WHO); 2008.

5. Ogden J. Psicologia da saúde. Lisboa: Climepsi Editores; 2004.

6. Sampaio D. Inventem-se novos pais. Lisboa: Editorial Caminho; 1994.

7. Sprinthall N, Collins W. Psicologia do Adolescente, uma abordagem desenvolvimentista. Lisboa: Fundação Calouste Gulbenkian; 2003.

8. World Health Organization (WHO). The Ottawa Charter for Health Promotion: First International Conference on Health Promotion, Ottawa, 21 November 1986. [página na Internet]. [acessado 2012 out 5]. Disponível em: http://www.who.int/healthpromotion/conferences /previous/ottawa/en/print.html

9. World Health Organization (WHO). Benefits of Physical Activity 2009. [página na Internet]. [acessado 2012 out 5]. Disponível em: http://www.who.int/dietphysical activity/factsheet_benefits/en/print.html

10. Cavill N, Kahlmeier S, Racioppi F. Physical activity and health in Europe: evidence for action. Copenhagen: World Health Organization (WHO); 2006.

11. Janssen I, Leblanc A. Systematic Review of the Health Benefits of Physical Activity in School-Aged Children and Youth. Int J Behav Nutr Phys Act 2010; 7:40.

12. Sibley B, Etnier J. The relationship between physical activity and cognition in children: a meta-analysis. Pediatr Exerc Sci 2003; 15:243-256.

13. Pate R, Trost S, Levin S, Dowda M. Sports participation and health-related behaviours among US youth. Arch Pediatr Adolesc Med 2000; 154(9):904-911.

14. Corte-Real N, Balaguer I, Dias C, Corredeira R, Fonseca A. Atividade física, prática desportiva, consumo de alimentos, de tabaco e de álcool dos adolescentes portugueses. Rev Port Sau Pub 2008; 26(2):17-25.

15. Matos M, Carvalhosa S, Diniz J. Factores associados à prática da actividade física nos adolescentes portugueses. Ana Psicológica 2002; 1(20):57-66.

16. Matos M, Equipa do Projecto Aventura Social \& Saúde. A saúde dos adolescentes portugueses: relatório do estudo HBSC 2010. Lisboa: Edições FMH; 2012.

17. Mota J, Sallis J. Actividade Física e saúde: factores de influência da actividade física nas crianças e adolescentes. Porto: Campo das Letras; 2002.

18. Shafey O, Eriksen M, Ross H, Mackay J. El atlas del tabaco - tercera edición. Atlanta: American Cancer Society; 2009. 
19. Matos M, Gaspar T, Vitória P, Clemente M. Comportamentos e atitudes sobre o tabaco em adolescentes portugueses fumadores. Psic Saúde \& Doenças 2003; 4(2):205-219.

20. Barreto S, Giatti L, Casado L, Moura L, Crespo C, Malta D. Exposição ao tabagismo entre escolares no Brasil. Cien Saude Colet 2010; 15(2):3027-3034.

21. Institute of Alcohol Studies. Alcohol and Health. St Ives: Institute of Alcohol Studies; 2008.

22. Morais C. Consumo de álcool e suas repercussões na Saúde Pública. In: Fundação da Juventude. Seminário Beber para esquecer ou beber para (sobre) viver - duas faces possiveis da mesma juventude. Porto: Fundação da Juventude; 1999. p. 33-43.

23. Moreira R. A adolescência e as bebidas alcoólicas. In: Fundação da Juventude. Seminário Beber para esquecer ou beber para (sobre)viver - duas faces possiveis da mesma juventude. Porto: Fundação da Juventude; 1999. p. 51-57.

24. Granville-Garcia A, Clementino M, Gomes M, Firmino R, Ribeiro G, Siqueira M. Alcohol consumption among adolescents: attitudes, behaviors and associated factors. Cien Saude Colet 2014; 19(1):7-16.

25. Diener Ed, Scollon C, Oiahi S, Dzokotom V, Suh E. Positivity And The Construction Of Life Satisfaction Judgments: Global Happiness Is Not The Sum Of Its Parts. J Happiness Stud 2000; 1:159-176.

26. Pavot W, Diener ED. Review of the Satisfaction With Life Scale. Psychological Assessment 1995; 5(2):164-172.

27. Bradley R, Corwyn R. Life satisfaction among European American, African American, Chinese American, Mexican American, and Dominican American adolescents. Int J Behav Dev 2004; 28(5):385-400.

28. Gilman R, Huebner E. Characteristics of Adolescents Who Report Very High Life Satisfaction. J Youth Adolesc 2006; 35(3):311-319.

29. Ferrão J. Portugal, três geografias em recombinação Espacialidades, mapas cognitivos e identidades territoriais. Lusotopie 2002; 2:151-158.

30. Portugal. Instituto de Desenvolvimento Rural e Hidráulica (IDRHd). Programa Nacional Leader +: Definição e caracterização da zona abrangida. Lisboa: IDRHd; 2000.

31. Alegria E. Litoral/Interior: clivagem com sentido? Variações populacionais inter-censitárias nas últimas duas décadas. II Congresso Português de Demografia 2004. [página na Internet]. [acessado 2013 jan 20]. Disponível em: http://www.apdemografia.pt/pdf_congresso/6

32. Instituto Nacional de Estatística (INE). Área Predominantemente Urbana 2009. [página na Internet]. [acessado 2013 jan 20]. Disponível em: http://metaweb. ine.pt/sim/conceitos/Detalhe.aspx?ID=PT\&cnc_co$\mathrm{d}=1070 \&$ cnc_ini $=17-12-2009$.
33. Instituto Nacional de Estatística (INE). Área Predominantemente Rural 2009. [página na Internet]. [acessado 2013 jan 20]. Disponível em: http://metaweb. ine.pt/sim/conceitos/Detalhe.aspx?ID=PT\&cnc_cod $=1084 \&$ cnc_ini $=17-12-2009$

34. Corte-Real N, Balaguer I, Fonseca A. Inventário de comportamentos relacionados com a saúde dos adolescentes. Porto: Gabinete de Psicologia do Desporto da Faculdade de Desporto da Universidade do Porto; 2004. (documento não publicado)

35. Diener E, Emmons R, Larsen R, Griffin S. The Satisfaction With Life Scale. J Pers Assess 1985; 49(1):71-75.

36. Santana P, Vaz A, Fachada M. O Estado de saúde dos portugueses. Uma perspectiva espacial. Rev. Estudos Demográficos 2004; 36:5-28.

37. Youngblade L, Curry L. The People They Know: Links Between Interpersonal Contexts and Adolescent Risky and Health-Promoting Behavior. Appl Dev Sci 2006; 10(2):96-106.

38. Mota J, Silva G. Association with socio-economic status and parental participation among a Portuguese sample. Sport, Education and Society 1999; 4(2):193-199.

39. Matos M, Simões C, Canha L, Fonseca S. Saúde e Estilos de Vida nos jovens portugueses - Relatório do estudo de 1996 da Rede Europeia HBSC/OMS. Lisboa: Edições FMH; 2000.

40. Fraga S, Sousa S, Santos A, Mello M, Lunet N, Padrão P, Barros H. Tabagismo em Portugal. Arq Med 2005; 19 (56):207-229

41. Fraga S, Ramos E, Barros H. Uso de tabaco por estudantes adolescentes portugueses e fatores associados. Rev Saude Publica 2006; 40(4):620-626.

42. Mendes V, Lopes P. Hábitos de consumo de álcool em adolescentes. Rev Toxicodependência 2007; 13(2):25-40.

43. Goldbeck L, Schmitz T, Besi T, Herschbach P, Henrich G. Life satisfaction decrease during adolescence. Qual Life Res 2007; 16(6):969-979.

44. Portugal. Ministério da Saúde. Plano Nacional de Saúde 2012-2016. [página na Internet]. [acessado 2013 abr 4]. Disponível em: http://pns.dgs.pt/pns-2012-2016

45. Portugal. Ministério da saúde. Programa Nacional de saúde Escolar. [página na Internet]. [acessado $2013 \mathrm{abr}$ 4]. Disponível em: http://www.min-saude.pt/NR/rdonlyres/4612A602-74B9-435E-B720-0DF22F70D36C/0/ ProgramaNacionaldeSa\%C3\%BAde Escolar.PDf

Artigo apresentado em 29/09/2013

Aprovado em 31/10/2013

Versão final apresentada em 07/11/2013 